\section{Lymphomatosis cerebri: a treatable cause of rapidly progressive dementia}

\section{INTRODUCTION}

Lymphomatosis cerebri (LC) is a rare variant of primary central nervous system lymphoma (PCNSL) pathologically characterised by diffuse cerebral infiltration of a non-cohesive mass of malignant lymphoid cells. We describe a case of rapidly progressive dementia where MRI demonstrated a diffuse leukoencephalopathy. After a series of normal investigations, brain biopsy was undertaken with histology consistent with LC. Our patient was successfully treated with chemoradiotherapy and neurorehabilitation. This case underlines the importance of timely brain biopsy in rapidly progressive cognitive decline to allow earlier therapy for potentially curable pathology.

\section{CASE REPORT}

A 50-year-old previously fit and well lady with no vascular risk factors presented to her general practitioner with personality change and emotional lability, against a background of subacutely progressive amnestic syndrome over 3 weeks. She next developed severe anxiety and insomnia and was commenced on citalopram and temazepam. Neurological examination demonstrated universally brisk reflexes with no clonus and downgoing plantar responses. There was marked language difficulty with both expressive and receptive components and ideational and ideomotor apraxia. Formal neuropsychometric testing showed marked impairment in memory, naming, literacy skills and information processing speed (all $<5$ th centile) with relative preservation of executive functioning (phonemic fluency 25th-50th centile). Testing was consistent with marked and pervasive cognitive dysfunction affecting mainly posterior, cortical and subcortical regions.

Full blood count, C-reactive protein, ESR, renal and liver function tests were normal. EEG showed left temporal irregular slowing with occasional low-voltage sharp waves suggestive of epileptiform activity. MRI examination (figure $1 \mathrm{~A}-\mathrm{C}$ ) demonstrated extensive confluent areas of T2-weighted hyperintensity diffusely throughout the cerebrum and brainstem. There was no perceptible mass effect, abnormal post-gadolinum contrast enhancement nor restriction of diffusion of the diffusion weighted imaging sequences.

The differential diagnoses included infective pathology such as JC virus and $\mathrm{HIV}$, rapidly progressive inflammatory demyelination and genetic metabolic causes. The following were normal or negative: JCV, HIV 1 and 2 and syphilis serology; plasma amino acids, white cell enzymes and very long chain fatty acids levels. Copper studies, coeliac screen, vitamin E levels, autoimmune antibody tests, serum lactate, pyruvate, phytanic and glutaric acid levels were also normal. Cerebrospinal fluid (CSF) examination showed a protein level of $0.74 \mathrm{~g} / \mathrm{L}$ with a glucose of $3.2 \mathrm{mmol} / \mathrm{L}$ (serum 5.5 $\mathrm{mmol} / \mathrm{L}),<1$ white blood cell per millilitre and negative oligoclonal bands. CSF PCR for viruses including JC and HIV were also negative. CSF cytology showed a moderately cellular fluid with increased numbers of lymphocytes and macrophages with no atypical cells, consistent with a mild chronic inflammatory/reactive process. Opthalmological screening revealed no evidence of vitreoretinal disease.

Her cognitive symptoms deteriorated over months to the point where she required $24 \mathrm{~h}$ nursing care due to significant confusion and anxiety. She became too unwell to engage in formal neuropsychometric tests, but two senior neurologists agreed that she had substantially deteriorated in posterior, cortical and subcortical domains compared with the aforementioned neuropsychometry. A repeat MRI (figure 1D-F) showed more extensive signal changes in the frontal, parietal, occipital and temporal lobes. This also involved the right corticospinal tract, resulting in symmetrical T2-weighted hyperintensity along the paired tracts through the capsular, mesencephalic, pontine and medullary regions. A further focus of T2weighted hyperintensity was seen in the left cerebellar hemisphere. There was now patchy enhancement in the peritrigonal white matter bilaterally.

Given the rapid clinicoradiological progression and lack of diagnosis, a brain biopsy of an enhancing region in the left parietal lobe was performed. Histological examination showed diffuse and nodular infiltration of cerebrum by large atypical lymphoid cells. The cells showed nuclear pleomorphism and showed clusters around blood vessels and diffuse spread into the surrounding cortex. Further tests confirmed a diffuse large $\mathrm{B}$ cell lymphoma.

Following a bone marrow biopsy and PET/CT scan, no evidence of lymphoma outside the central nervous system was found. Treatment commenced, including high-dose methotrexate, cytarabine and rituximab, followed by radiotherapy (40 Gy total). Repeat MRI showed significant improvement in the central nervous system disease (figure 1G-I) and she also improved functionally, being discharged home without a package of care after previously needing $24 \mathrm{~h}$ nursing care. Ten months later, functional status had not deteriorated and neuropsychometric testing was equivalent to that done several months prior to commencement of treatment (ie, before significant further cognitive decline), however remaining below premorbid estimates. Importantly, her gait and mobility also improved dramatically as she went from being unable to walk unaided immediately prior to treatment, to walking independently, swimming twice weekly and using a cross-trainer regularly.

\section{DISCUSSION}

PCNSL accounts for 3\% of primary central nervous system tumours. ${ }^{1}$ It typically presents as multifocal T2 hyperintense lesions that both enhance after gadolinium administration and demonstrate mass effect. A case study in 1999 coined the term 'lymphomatosis cerebri' to describe an exceedingly rare variant of PCNSL characterised by diffuse parenchymal infiltration of lymphomatous cells. ${ }^{2}$ Previous presentations include gait disturbance (mainly ataxic), personality change, memory deficits and weight loss. ${ }^{3}$

White matter abnormalities in LC have been described affecting all regions of the brain; however, serial MRI has rarely been performed. We demonstrated rapid progression of radiological features that temporally correlated with clinical decline. We also demonstrated transition from nonenhancing to enhancing lesions perhaps suggesting that progression and evolution of the disease is associated with eventual disruption of the blood-brain barrier.

Where LC was diagnosed premortem, many cases responded to steroids alone, at least initially. ${ }^{4}$ To achieve complete remission, steroid use has been followed by radiotherapy, cisplatin or methotrexate. ${ }^{4}$ 

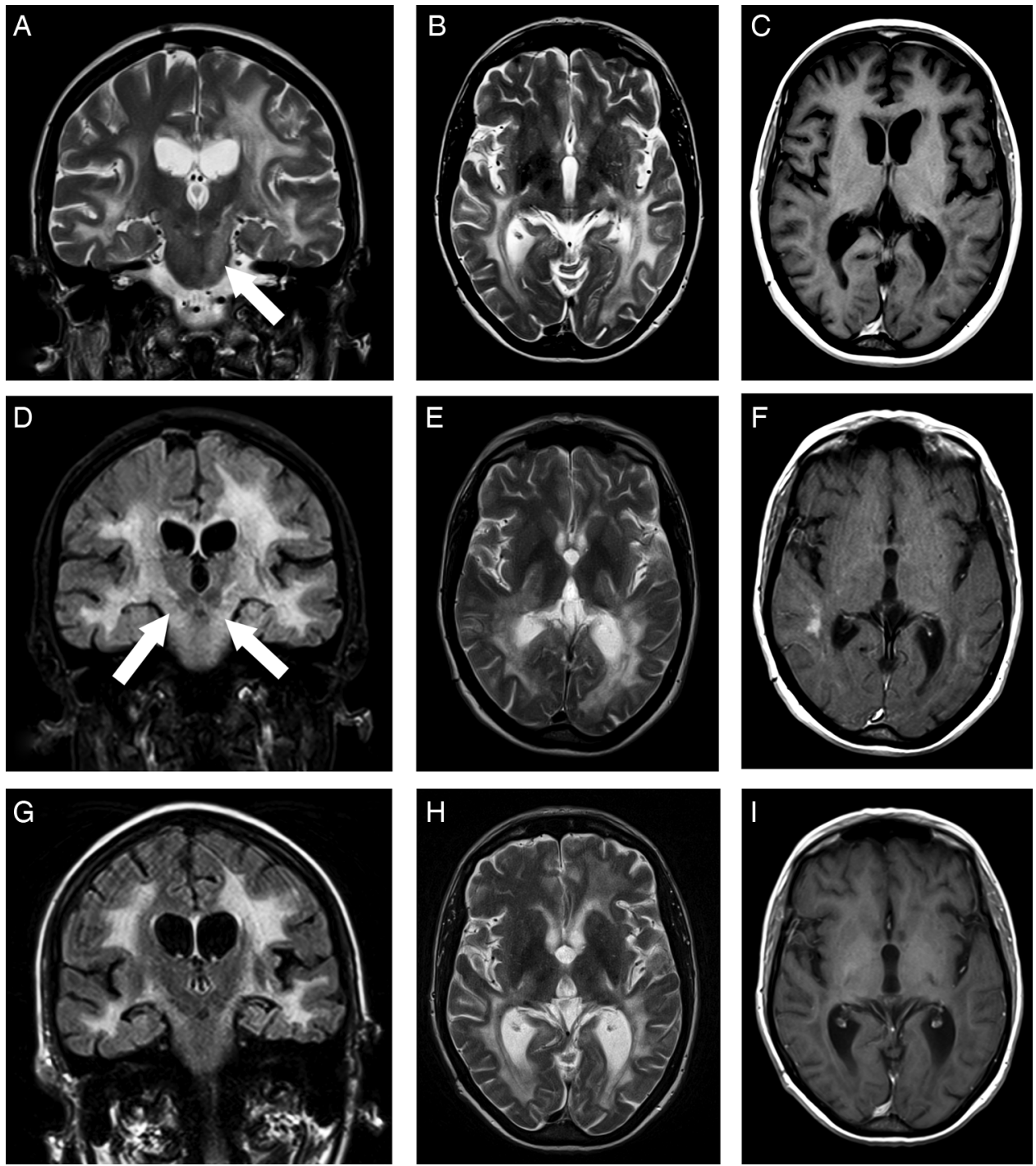

Figure 1 Brain magnetic resonance imaging (MRI) at presentation $(A-C)$, with coronal $(A)$ and axial (B) T2-weighted sequences demonstrating extensive white matter hyperintensity with no associated mass effect. Note the extension of the hyperintensity along the left corticospinal tract at the level of the cerebral peduncle and pons (white arrow). Axial post-gadolinium contrast T1-weighted imaging (C) did not reveal any abnormal enhancement at this stage. Brain MRI at 6 months after initial presentation (D-F) with coronal FLAIR (D) and axial T2-weighted (E) sequences demonstrating progression and some mass effect of the white matter signal abnormality now involving both corticospinal tracts at the mesencephalic and pontine levels (white arrows). The axial post-gadolinium contrast T1-weighted (F) imaging shows patchy peritrigonal white matter enhancement. Follow-up MRI examination post-therapy $(\mathrm{G}-\mathrm{I})$ with coronal FLAIR $(\mathrm{G})$ and axial T2-weighted (H) sequences demonstrating an improvement in the extent and mass effect of the white matter hyperintensity and the resolution of peritrigonal white matter enhancement on the axial post-gadolinium contrast T1-weighted (I) imaging.

There are many causes of rapidly progressive leukoencephalopathy including inflammatory, vascular, immune, infectious, neoplastic, drug and toxic causes, neurodegenerative conditions and rarely genetic leukoencephalopathy, for example, cerebral adrenoleukodystrophy.

Nonetheless, rapid progression of structural change, perhaps combined with new foci of enhancement, should prompt thoughts of an underlying neoplastic process and brain biopsy. A recent review addressing the approach to diagnosing adult onset leukodystrophy suggests the diagnostic yield of biopsy is up to $78 \%$ with management being altered in $63 \%$ of cases. ${ }^{5}$ This case highlights the diagnostic (and potential therapeutic) importance of early consideration of brain biopsy in patients with rapidly progressive atypical neurological syndromes including cognitive decline.

Amit Samani, ${ }^{1}$ Indran Davagnanam, ${ }^{2}$ Oliver Charles Cockerell, ${ }^{3}$ Alan Ramsay, ${ }^{4}$ Rickie Patani, ${ }^{15,6,7}$ Jeremy Chataway ${ }^{1}$

${ }^{1}$ Department of Neuroinflammation, The National Hospital for Neurology and Neurosurgery, London, UK ${ }^{2}$ Brain Repair and Rehabilitation Unit, UCL Institute of Neurology, London, UK

${ }^{3}$ Department of Neurology, The Wellington Hospital, London, UK

${ }^{4}$ Department of Cellular Pathology, University College London Hospital, London, UK
${ }^{5}$ Department of Molecular Neuroscience, Institute of Neurology, University College London, London, UK ${ }^{6}$ Department of Clinical Neurosciences, University of Cambridge, Cambridge, UK

'University of Edinburgh, Edinburgh, UK

Correspondence to Dr Jeremy Chataway, Department of Neuroinflammation, Queen Square MS Centre, The National Hospital for Neurology and Neurosurgery, Queen Square, London WC1 3BG, UK; jeremy. chataway@uclh.nhs.uk

Contributors $A S, R P, I D, O C C$ and JC were involved in writing the manuscript. OCC and JC were the patient's consultant neurologists, and OCC, JC and RP were involved in the management of the patient. ID was involved in the interpretation and formatting of the diagnostic imaging panels. AR reported and supplied the pathology. 
Competing interests RP is a Wellcome Trust Clinician Scientist and an Anne Rowling Fellow in Regenerative Neurology.

Provenance and peer review Not commissioned; externally peer reviewed.

$\mathrm{RP}$ and JC contributed equally.

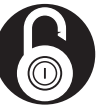

\section{OPEN ACCESS}

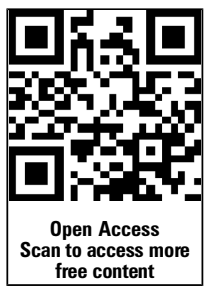

Open Access This is an Open Access article

distributed in accordance with the Creative Commons Attribution Non Commercial (CC BY-NC 3.0) license, which permits others to distribute, remix, adapt, build upon this work non-commercially, and license their derivative works on different terms, provided the original work is properly cited and the use is noncommercial. See: http://creativecommons.org/licenses/ by-nc/3.0/

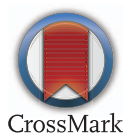

To cite Samani A, Davagnanam I, Cockerell O C, et al. J Neurol Neurosurg Psychiatry 2015;86:238-240.

Received 28 November 2013

Revised 15 April 2014

Accepted 27 April 2014

Published Online First 20 May 2014

J Neurol Neurosurg Psychiatry 2015;86:238-240.

doi:10.1136/jnnp-2013-307327

\section{REFERENCES}

1 Gestner ER, Batchelor TT. Primary Central Nervous System Lymphoma. Arch Neurol 2010;67:291-7.

2 Bakshi R, Mazziotta JC, Mischel PS, et al. Lymphomatosis cerebri presenting as a rapidly progressive dementia: clinical, neuroimaging and pathologic findings. Dement Geriatr Cogn Disord 1999;10:152-7.

3 Kanai $R$, Shibuya M, Hata T, et al. A case of 'lymphomatosis cerebri' diagnosed in an early phase and treated by whole brain radiation: case report and literature review. J Neurooncol 2008;86: 83-8.

4 Leschziner G, Rudge P, Lucas S, et al. Lymphomatosis cerebri presenting as a rapidly progressive dementia with a high methylmalonic acid. J Neurol 2011;258: 1489-93.

5 Ahmed RM, Murphy E, Davagnanm I, et al. A practical approach to diagnosing adult onset leukodystrophy. J Neurol Neurosurg Psychiatry 2014;85:770-81. 